\title{
Constraints on Axionlike Particles from a Hard X-Ray Observation of Betelgeuse
}

\author{
Mengjiao Xiao $\odot,{ }^{1, *}$ Kerstin M. Perez, ${ }^{1, \dagger}$ Maurizio Giannotti $\odot,{ }^{2, \ddagger}$ Oscar Straniero, ${ }^{3, \S}$ Alessandro Mirizzi, ${ }^{4,5, \|}$ \\ Brian W. Grefenstette, ${ }^{6, \pi}$ Brandon M. Roach $\oplus^{1, * *}$ and Melania Nynka, ${ }^{7, \dagger}$ \\ ${ }^{1}$ Department of Physics, Massachusetts Institute of Technology, Cambridge, Massachusetts 02139, USA \\ ${ }^{2}$ Physical Sciences, Barry University, 11300 NE 2nd Ave., Miami Shores, Florida 33161, USA \\ ${ }^{3}$ Istituto Nazionale di Astrofisica, Osservatorio Astronomico d'Abruzzo, 64100 Teramo, Italy \\ ${ }^{4}$ Dipartimento Interateneo di Fisica "Michelangelo Merlin", Via Amendola 173, 70126 Bari, Italy \\ ${ }^{5}$ Istituto Nazionale di Fisica Nucleare-Sezione di Bari, Via Orabona 4, 70126 Bari, Italy \\ ${ }^{6}$ Cahill Center for Astrophysics, 1216 E. California Blvd, California Institute of Technology, Pasadena, California 91125, USA \\ ${ }^{7}$ MIT Kavli Institute for Astrophysics and Space Research, 77 Massachusetts Avenue, Cambridge, Massachusetts 02139, USA
}

(Received 14 October 2020; revised 28 November 2020; accepted 11 December 2020; published 21 January 2021)

\begin{abstract}
We use the first observation of Betelgeuse in hard x rays to perform a novel search for axionlike particles (ALPs). Betelgeuse is not expected to be a standard source of $\mathrm{x}$ rays, but light ALPs produced in the stellar core could be converted back into photons in the Galactic magnetic field, producing a detectable flux that peaks in the hard x-ray band $\left(E_{\gamma}>10 \mathrm{keV}\right)$. Using a $50 \mathrm{ks}$ observation of Betelgeuse by the NuSTAR satellite telescope, we find no significant excess of events above the expected background. Using models of the regular Galactic magnetic field in the direction of Betelgeuse, we set a 95\% C.L. upper limit on the ALP-photon coupling of $g_{a \gamma}<(0.5-1.8) \times 10^{-11} \mathrm{GeV}^{-1}$ (depending on magnetic field model) for ALP masses $m_{a}<(5.5-3.5) \times 10^{-11} \mathrm{eV}$.
\end{abstract}

DOI: $10.1103 /$ PhysRevLett.126.031101

Introduction.-Axionlike particles (ALPs) are ultralight pseudoscalar bosons with a two-photon vertex $g_{a \gamma}$, predicted by several extensions of the standard model (see Refs. [1,2] for a recent review). In the presence of an external magnetic field, the $g_{a \gamma}$ coupling leads to the phenomenon of photon-ALP mixing [3]. This effect is exploited by several ongoing and upcoming ALP search experiments (see Refs. [1,4,5] for recent reviews).

The photon-ALP coupling would also cause ALPs to be produced in stellar plasmas via the Primakoff process [6]. Therefore astrophysical observations offer unique sensitivity to ALPs. Analyses of the lifetime of helium-burning stars in globular clusters have excluded the couplings $g_{a \gamma}>6 \times 10^{-11} \mathrm{GeV}^{-1} \quad\left(95 \% \quad\right.$ C.L.) for $m_{a} \lesssim 30 \mathrm{keV}$ [7-9]. This is the strongest bound on the ALP-photon coupling in a wide mass range. A comparable bound, $g_{a \gamma}>$ $6.6 \times 10^{-11} \mathrm{GeV}^{-1}$ (95\% C.L.) for $m_{a} \lesssim 0.02 \mathrm{eV}$, was derived by the CAST experiment, which searches for ALPs produced in the solar core that are reconverted into $\mathrm{x}$ rays in a large laboratory magnetic field [10].

At lower ALP masses, as shown in Fig. 1, more stringent bounds are derived from supernovae and galaxy cluster observations. A recent reanalysis of the SN 1987A limit, derived from the nonobservation of gamma rays induced by conversion in the Galactic magnetic field of ALPs produced in the supernova core, excludes $g_{a \gamma}>5.3 \times 10^{-12} \mathrm{GeV}^{-1}$ for $m_{a}<4.4 \times 10^{-10} \mathrm{eV}$ (95\% C.L.) [11]. A search for gamma-ray bursts from extragalactic supernovae with the Fermi large area telescope (LAT) has yielded a weaker limit, $g_{a \gamma} \lesssim 2.6 \times 10^{-11} \mathrm{GeV}^{-1}$ for ALP masses $m_{a} \lesssim 3 \times$ $10^{-10} \mathrm{eV}$ [12], under the assumption of at least one supernova occurring in the LAT field of view. However, the SN 1987A bound has been questioned due to uncertainties on the response of the GRS instrument on the SMM satellite [13], and both of these bounds are subject to uncertainties due to the modeling of core-collapse supernovae and Galactic magnetic fields. The most stringent limits to date on low-mass ALPs come from the search for

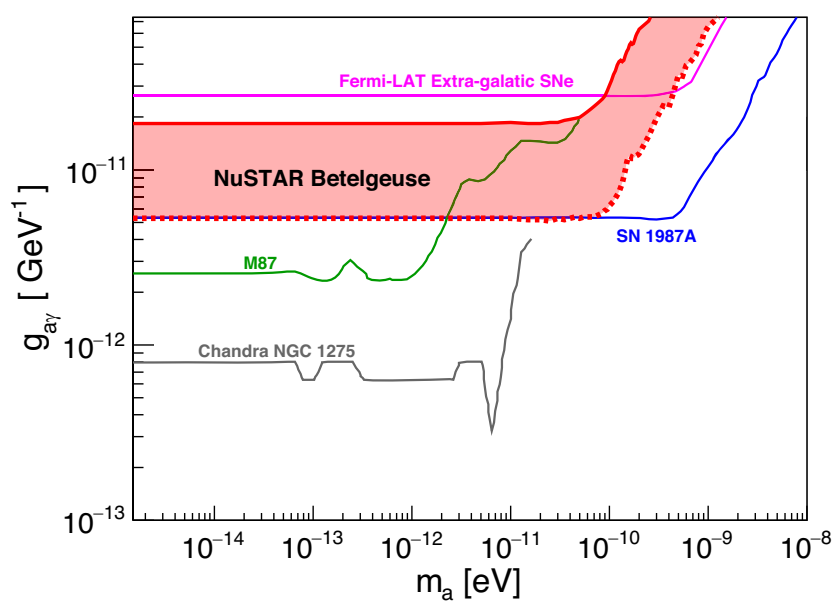

FIG. 1. Astrophysical constraints on the ALP-photon coupling in relation to the Betelgeuse bound from the present work: SN 1987A [11], Fermi-LAT extragalactic SNe [12], M87 [16], and Chandra NGC 1275 [14]. 
$\mathrm{x}$-ray spectral distortions in the active galactic nucleus NGC 1275 at the center of the Perseus cluster, induced by conversion in the intracluster medium. These exclude $g_{a \gamma}>$ $(6-8) \times 10^{-13} \mathrm{GeV}^{-1}$ (99.7\% C.L.) for $m_{a}<10^{-12} \mathrm{eV}$ [14]. However, these results could be weakened by several orders of magnitude if the intracluster magnetic field has been mismodeled [15].

Here, we exploit the fact that Betelgeuse, a nearby red supergiant star, provides an excellent laboratory for ALPs, as proposed in a seminal paper by Carlson [17]. Betelgeuse ( $\alpha$ Orionis, spectral type M2Iab) is an ideal candidate for ALP searches, as it (i) has a hot core, and thus is potentially a copious producer of ALPs that, after reconversion in the Galactic magnetic field, produces a photon signal peaked in the hard x-ray range, and (ii) is in region of the Hertzsprung-Russel diagram where no stable corona is expected, and thus has essentially zero standard astrophysical x-ray background [18]. This basic idea can be extended to other stellar objects, such as clusters of hot, young stars [19]. Betelgeuse has the additional advantage that it is nearby, at a distance $d \sim 200 \mathrm{pc}[20,21]$, and thus in a region of the local magnetic field that is relatively easier to constrain with future observations.

In this work, we use a dedicated observation of Betelgeuse by the NUSTAR satellite telescope to derive limits on the ALP-photon coupling $g_{a \gamma}<(0.5-1.8) \times 10^{-11} \mathrm{GeV}^{-1}$ (95\% C.L.) for masses $m_{a}<(5.5-3.5) \times 10^{-11} \mathrm{eV}$ (depending on the assumed value of the regular Galactic magnetic field). We derive a range of ALP spectra expected from Betelgeuse, depending on its precise evolutionary stage. We then derive the expected $\mathrm{x}$-ray spectra after conversion in the regular Galactic magnetic field, and compare to our spectral measurement in the energy range 3-79 keV. For low-mass ALPs, these limits are a factor of $\sim 3$ deeper than the limits from CAST, and are comparable to the limits expected from next-generation ALP experiments such as ALPS-II [22] and BabyIAXO [23,24]. They are competitive with the constraints from SN 1987A, though with independent sources of systematic uncertainty. We emphasize that the preponderance of astrophysical exclusions that overlap in this region of $g_{a \gamma}-m_{a}$ parameter space, each with a separate modeling assumptions and sources of uncertainty, builds confidence in the robustness of these constraints.

ALP and photon fluxes from Betelgeuse.-In the minimal scenarios, ALPs have only a two-photon coupling, described by the Lagrangian [3],

$$
\mathcal{L}_{a \gamma}=-\frac{1}{4} F_{\mu \nu} \tilde{F}^{\mu \nu} a=\mathbf{E} \cdot \mathbf{B} a .
$$

This interaction allows for ALP production in a stellar medium primarily through the Primakoff process, in which thermal photons are converted into ALPs in the electrostatic field of ions, electrons, and protons. The ALP production rate via the Primakoff process in a stellar core can be calculated as (see, e.g., Ref. [17]),

$$
\begin{aligned}
\frac{d \dot{n}_{a}}{d E}= & \frac{g_{a \gamma}^{2} \xi^{2} T^{3} E^{2}}{8 \pi^{3}\left(e^{E / T}-1\right)} \\
& \times\left[\left(1+\frac{\xi^{2} T^{2}}{E^{2}}\right) \ln \left(1+\frac{E^{2}}{\xi^{2} T^{2}}\right)-1\right],
\end{aligned}
$$

where $E$ is the photon energy, $T$ the temperature, and $\xi^{2}=\kappa^{2} / 4 T^{2}$ with $\kappa$ the inverse of the screening length, introduced by the finite range of electric field surrounding charged particles in the plasma. Once produced, ALPs can easily escape the star since their mean free path in stellar matter is sufficiently large for the values of mass and coupling we are interested in. The total ALP spectrum can then be obtained by integrating Eq. (2) over the volume of the star, $d \dot{N}_{a} / d E=\int\left(d \dot{n}_{a} / d E\right) d V$, which can be well parametrized by fitting the stellar model (see Supplemental Material [25] for details).

The ALP spectrum thus depends on the physical structure and chemical evolution of the star. Betelgeuse, due to its relative proximity, has fairly well-constrained observed values of luminosity, effective temperature, and metallicity (see Supplemental Material [25]). However, the time until core collapse is not well known, and as the temperature and density of the core increase as the star approaches supernova, this introduces significant uncertainty on the predicted ALP spectra. We use the full network stellar evolution code (FuNS [35]) to derive 13 models of the Betelgeuse ALP source spectrum, parametrized by the time until core collapse, $t_{\mathrm{cc}}$. These range from an optimistic ALP production scenario $\left(t_{\mathrm{cc}}=1.4 \mathrm{yr}\right)$ to a conservative ALP flux scenario $\left(t_{\mathrm{cc}}=1.55 \times 10^{5} \mathrm{yr}\right)$, as shown in Table $\mathrm{S} 1$ of the Supplemental Material [25].

The interaction in Eq. (1) may also trigger ALP-photon oscillations in external magnetic fields, such as those found in our galaxy, thus producing a detectable x-ray flux. The calculation of the ALP-photon reconversion probability simplifies if we restrict ourselves to the case in which $\mathbf{B}$ is homogeneous. The magnetic field of the Galaxy is known to change on scales of $\sim 1 \mathrm{kpc}$, corresponding to the arm and interarm regions, and between the Galactic disk and halo [36-38]. Motivated by the relative proximity of Betelgeuse in the Galactic disk, we assume conversion in a homogenous regular magnetic field. Although the magnetic structure of the Galaxy is certainly more complex -including turbulent fields with coherent lengths $\mathcal{O}(200)$ or smaller [38-40], such as due to supernovae, molecular clouds, and our own Local Bubble-this assumption follows the convention of previous astrophysical bounds, e.g., SN 1987A [11]. This allows for consistent comparison between constraints. A detailed treatment of the effect of magnetic field correlation lengths and amplitudes on the conversion probability is deferred to a later dedicated theoretical study.

In this framework, the differential photon flux per unit energy arriving at Earth is 


$$
\frac{d N_{\gamma}}{d E d S d t}=\frac{1}{4 \pi d^{2}} \frac{d \dot{N}_{a}}{d E} P_{a \gamma}
$$

The ALP-photon conversion probability is [41]

$$
P_{a \gamma}=8.7 \times 10^{-6} g_{11}^{2}\left(\frac{B_{T}}{1 \mu \mathrm{G}}\right)^{2}\left(\frac{d}{197 \mathrm{pc}}\right)^{2} \frac{\sin ^{2} q}{q^{2}},
$$

where $B_{T}$ is the transverse magnetic field, namely, its component in the plane normal to the path between Earth and Betelgeuse, $q$ is the momentum transfer, and $d$ is the magnetic field length.

$$
\begin{aligned}
q \simeq & {\left[77\left(\frac{m_{a}}{10^{-10} \mathrm{eV}}\right)^{2}-0.14\left(\frac{n_{e}}{0.013 \mathrm{~cm}^{-3}}\right)\right] } \\
& \times\left(\frac{d}{197 \mathrm{pc}}\right)\left(\frac{E}{1 \mathrm{keV}}\right)^{-1},
\end{aligned}
$$

$n_{e}$ is the electron density [42]. Practically, the differential photon flux per unit energy arriving at Earth can be numerically calculated by Eq. (S2) where the parameters are fitted with stellar evolution model and listed in Table S1. The predicted ALP-photon spectra are illustrated in Fig. S2 of the Supplemental Material [25].

For small values of $q$ (small ALP mass) and our assumed homogenous B field, the expected ALP flux does not depend strongly on the distance to the source, as the drop in flux with distance is compensated by an increase in the conversion probability [Eqs. (4)-(5)]. Thus we ignore any uncertainty due to the distance to Betelgeuse for the small ALP masses considered here. For large $q$ (for large ALP mass), the increase in the conversion probability with the distance is lost because of incoherence effects.

Even within the simplifying assumption of a uniform regular magnetic field, the exact value of the magnetic field in the direction of Betelgeuse introduces a considerable source of uncertainty in our estimate of the x-ray flux. The reported values of the local regular magnetic field, translated to $B_{T}$ in the direction of Betelgeuse, vary between 0.4 [37] and 3.0 $\mu \mathrm{G}$ [43]. Here we are using $1.4 \mu \mathrm{G}$ [38] as a representative value and 0.4 and $3.0 \mu \mathrm{G}$ as the lower and upper bound.

Spectral analysis.-The NuSTAR observatory [44-47] is the first focusing high-energy $\mathrm{x}$-ray telescope in orbit. Its 3-79 keV energy range is ideal for probing ALP signals from Betelgeuse. NUSTAR has two identical co-aligned telescopes, each consisting of an independent optic and focal-plane detector, referred to as FPMA and FPMB. Each telescope subtends a field of view of approximately $13^{\prime} \times 13^{\prime}$, with a half-power diameter of $\sim 60^{\prime \prime}$ for a point source near the optical axis.

We use a dedicated NUSTAR observation of Betelgeuse taken on 23 August 2019 (ObsID 30501012002). We processed the data using the standard NUSTAR data reduction pipeline, NUSTARDAS V1.8.1 distributed in HEASOFT v6.24, and the latest calibration package (CALDB. indx20191219). We used the flags SAAMODE=OPTIMIZED and TENTACLE=YES to exclude time intervals with elevated instrument backgrounds, coinciding with the telescope passing through the South Atlantic anomaly (SAA). After this filtering, the total cleaned exposure was $49.2 \mathrm{ks}$ for FPMA, and 48.4 ks for FPMB.

We extract spectra with NUPRODUCTS, using a circular source region of radius $60^{\prime \prime}$ around the star's equatorial coordinates (RA 88.79293', Dec. 7.40706 ${ }^{\circ}$ ) [48]. We simultaneously extract the instrument response files used to generate the ALP signal spectra that would be observed in this same region, in particular the auxiliary response file (ARF) which defines the energy-dependent effective area for this source region, and the redistribution matrix file (RMF) which contains the detector quantum efficiency and energy resolution [44].

We extract background spectra from nearby regions, as shown in Fig. S3 of Ref. [25]. These regions are separated from the center of the source region by at least $120^{\prime \prime}$ in order to ensure that contamination from the source region is below the level of $\mathcal{O}(1 \%)$. In addition, we observe a point source near the edge of both FPMs, whose position is consistent with Chandra source CXO J055520.2 + 072002 [49]. This source is not detected above $10 \mathrm{keV}$, but to be conservative we choose the background region to be at least $60^{\prime \prime}$ from this object. The background region is chosen to be on the same detector chip as the source region, in order to properly describe any spatially varying backgrounds. In particular, the NUSTAR instrumental background, including $\mathrm{x}$-ray lines resulting from fluorescence or activation of the instrument materials, is known to vary between detector chips, and the stray light from the cosmic x-ray background varies radially along the detector plane [50]. Our results are robust to alternate choices of background region, as shown in the Supplemental Material [25].

The observed x-ray spectra for FPMA and FPMB in the source region and background region are shown in the top panel of Fig. 2. Both source and background rates are higher for FPMB than for FPMA, due to the higher instrumental background in this detector. The background-subtracted source spectra are shown in the lower panel of Fig. 2. These were prepared by normalizing the background spectra by the area of the source extraction region, following the procedure in NUPRODUCTS. After background subtraction, the source spectrum fluctuates around zero counts. We confirm that our upper limit on the background-subtracted count rate from Betelgeuse is consistent with that measured in soft $\mathrm{x}$ rays $(0.3-8 \mathrm{keV})$ using data from Chandra (see the Supplemental Material [25]).

The source spectra after the background subtraction is compared to examples of the predicted $\mathrm{x}$-ray spectra from ALP production, for the case of $B_{T}=1.4 \mu \mathrm{G}$, $m_{a}=10^{-11} \mathrm{eV}$, and $g_{a \gamma}=1.5 \times 10^{-11} \mathrm{GeV}^{-1}$, also shown in the lower panel of Fig. 2. We numerically calculate the 


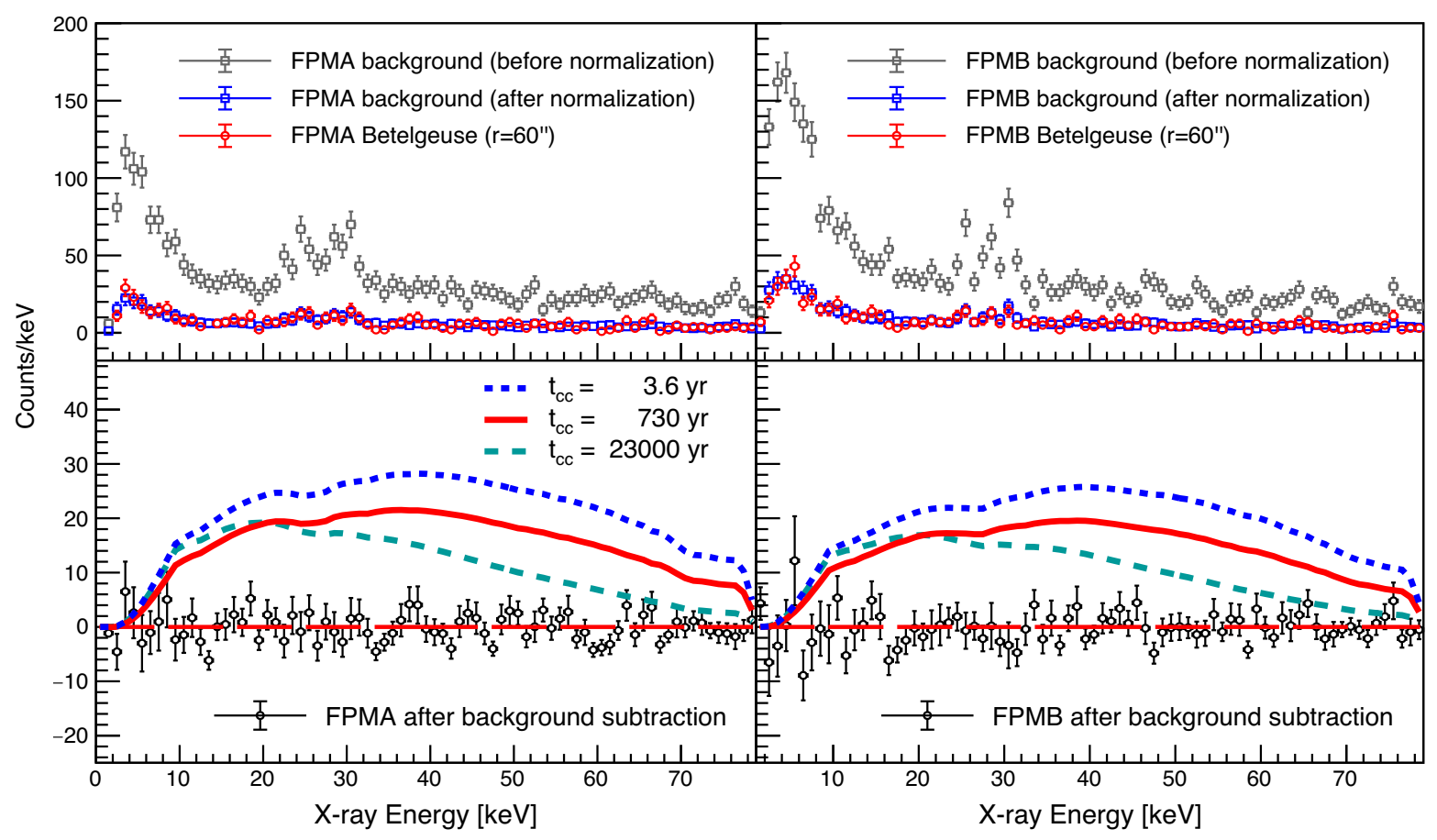

FIG. 2. Top: x-ray spectra from FPMA (left) and FPMB (right) for the Betelgeuse source (red) and background (gray and blue for before and after normalization) regions. The error bars overlaid are the statistic uncertainties $(\sqrt{N})$. Bottom: Source spectra after subtracting the normalized background. The error bars are calculated by Sumw2 with ROOT software [51]. The predicted ALP-produced $\mathrm{X}$-ray spectra assuming transverse magnetic field $B_{T}=1.4 \mu \mathrm{G}$, mass $m_{a}=10^{-11} \mathrm{eV}$, and coupling $g_{a \gamma}=1.5 \times 10^{-11} \mathrm{GeV}^{-1}$, that would be detected by the NuSTAR instrument are overlaid. The stellar model parameters are described in Table S1 [25]. The spectra are binned to a width of $1 \mathrm{keV}$, though analysis is performed on unbinned data.

ALP-produced photon spectrum $d N_{\gamma} / d E_{\gamma}$ that would be detected by NUSTAR by folding the predicted spectrum from Eq. (S2) through the instrument response files extracted by NUPRODUCTS for this source region.

Data analysis and results. - Based on the predicted number of photons from ALPs $\left(N_{\mathrm{ax}}\right)$ and the expected number of background events $\left(N_{\mathrm{bkg}}\right)$ in our source region, we first optimized the energy range used for our analysis by maximizing the figure-of-merit $N_{\mathrm{ax}} / \sqrt{N_{\mathrm{bkg}}}$. This was done before inspecting our source data. Because of the difference in shape for the predicted ALP spectra, we use three different energy ranges: $10-60 \mathrm{keV}$ for the model with $t_{\mathrm{cc}}=1.55 \times 10^{5} \mathrm{yr}, 10-70 \mathrm{keV}$ for $t_{\mathrm{cc}}=6900-23000 \mathrm{yr}$, and $10-79 \mathrm{keV}$ for $t_{\mathrm{cc}}=1.4-3700 \mathrm{yr}$. The number of source events $\left(N_{\mathrm{obs}}\right)$ and expected background events $\left(N_{\mathrm{bkg}}\right)$ in the optimized energy ranges for FPMA and FPMB are listed in Table I. The observed events in the Betelgeuse source region are consistent with the expected background events within the statistic uncertainties for both FPMA and FPMB.

To fit the data, an unbinned likelihood function is constructed as [52]

$$
\mathcal{L}=\prod_{i=1}^{n} \mathcal{L}_{i} \times \prod_{i=1}^{n} \operatorname{Gauss}\left(\delta_{\mathrm{bkg}}^{i}, \sigma_{\mathrm{bkg}}^{i}\right)
$$

where

$$
\begin{aligned}
\mathcal{L}_{i}= & \operatorname{Poisson}\left(N_{\mathrm{obs}} \mid N_{\mathrm{exp}}\right) \\
& \times \prod_{j=1}^{N_{\mathrm{obs}}}\left[\frac{N_{\mathrm{ax}} P_{\mathrm{ax}}\left(E_{\gamma}^{j}\right)}{N_{\exp }}+\frac{N_{\mathrm{bkg}}\left(1+\delta_{\mathrm{bkg}}\right) P_{\mathrm{bkg}}\left(E_{\gamma}^{j}\right)}{N_{\exp }}\right] .
\end{aligned}
$$

Here, $N_{\text {obs }}$ is the total number of events observed in our source region, and $N_{\text {exp }}=N_{\mathrm{ax}}+N_{\mathrm{bkg}}\left(1+\delta_{\mathrm{bkg}}\right)$ is the total number of events expected in our source region for the case of an ALP signal. $P_{\mathrm{ax}}\left(E_{\gamma}\right)$ is the energy-dependent ALP signal PDF, defined for given $m_{a}, g_{a \gamma}, t_{\mathrm{cc}}$, and $B_{T}$ (examples are shown in the lower panel of Fig. 2 and Fig. S2 of the Supplemental Material [25]). $P_{\mathrm{bkg}}\left(E_{\gamma}\right)$ is the background PDF, obtained by normalizing the background spectrum to the source region size using NUPRODUCTS, as

TABLE I. Observed events in the source region and expected background events, after normalization to the source region area, for FPMA and FPMB.

\begin{tabular}{lccccc}
\hline \hline & \multicolumn{2}{c}{ FPMA } & & \multicolumn{2}{c}{ FPMB } \\
\cline { 2 - 3 } \cline { 5 - 6 } Photon energy & $N_{\text {obs }}$ & $N_{\text {bkg }}$ & & $N_{\text {obs }}$ & $N_{\text {bkg }}$ \\
\hline $10-60 \mathrm{keV}$ & 313 & 315.8 & & 352 & 362.7 \\
$10-70 \mathrm{keV}$ & 354 & 359.8 & & 397 & 406.4 \\
$10-79 \mathrm{keV}$ & 384 & 392.7 & & 433 & 441.2 \\
\hline \hline
\end{tabular}




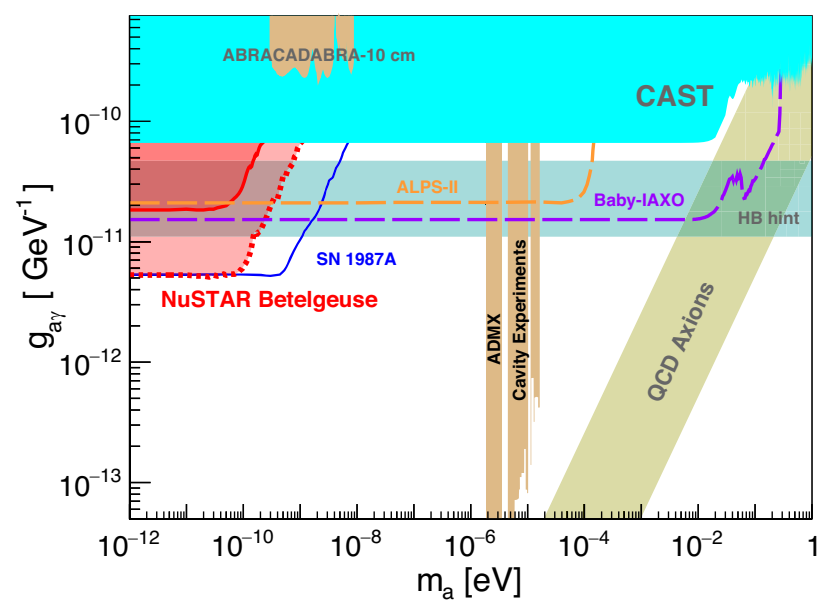

FIG. 3. Comparison of $95 \%$ upper limits of $g_{a \gamma}$ from NuSTAR Betelgeuse observation is shown in the red band: upper solid line for the most conservative stellar model and $B_{T}$, lower dashed line for the most optimistic stellar model and $B_{T}$, and the interval for the other combinations. Overlaid are the region of interest for the QCD axion [55], the current limits from CAST [10], ADMX [56], ABRACADABRA-10 cm [57], and SN 1987A [11], the projected sensitivity of ALPS-II [22] and Baby-IAXO [23,24]. Also labeled are the ALP regions suggested by hints of excess gammaray transparency or cooling of horizontal-branch stars [58,59].

described above. $\delta_{\mathrm{bkg}}$ and $\sigma_{\mathrm{bkg}}$ are the nuisance parameter and fractional systematic uncertainty of the background; $\operatorname{Gauss}\left(\delta_{\mathrm{bkg}}, \sigma_{\mathrm{bkg}}\right)$ is the Gaussian penalty term. Given the statistics of expected background events in the observation region, $\sigma_{\mathrm{bkg}}$ is set at $10 \%$ for both FPMA and FPMB, but allowed with independent Gaussian fluctuation.

The standard profile likelihood test statistic $[53,54]$ is used to derive constraints on the ALP-photon coupling $g_{a \gamma}$. The test statistic $q$ is defined as

$$
q\left(g_{\text {test }}\right)= \begin{cases}-2 \ln \frac{\mathcal{L}_{\max }\left(g_{\text {test }}, \dot{\theta}\right)}{\mathcal{L}_{\text {max }}\left(g_{\text {best }}, \hat{\theta}\right)}, & g_{\text {test }} \geq g_{\text {best }} \\ 0, & g_{\text {test }}<g_{\text {best }}\end{cases}
$$

For each choice of $m_{a}, t_{\mathrm{cc}}$, and $B_{T}$, we scan through the ALP-photon coupling $g_{\text {test }}$, and perform two maximum likelihood fits, one with the $g_{a \gamma}$ as its best fit value $g_{\text {best }}$, and the other with $g_{a \gamma}$ fixed at $g_{\text {test }}$. The nuisance parameters are all allowed to vary in both to achieve the best fit. We derive the $95 \%$ C.L. upper limit on $g_{a \gamma}$ assuming $q\left(g_{\text {test }}\right)$ follows a half- $\chi^{2}$ distribution with a single degree of freedom [53].

The main sources of uncertainty on the ALP signal are the choice of stellar model $\left(t_{\mathrm{cc}}\right)$ and assumed value of the transverse local Galactic magnetic field strength $\left(B_{T}\right)$. Rather than accounting for these as nuisance parameters in the likelihood function, we derive separate $95 \%$ C.L. limits on $g_{a \gamma}$ for each of the 13 stellar models for each of our three assumed values of $B_{T}$.

The final $95 \%$ C.L. upper limit on $g_{a \gamma}$ is shown in Fig. 3 and Fig. 1. The region labeled in red is excluded by this

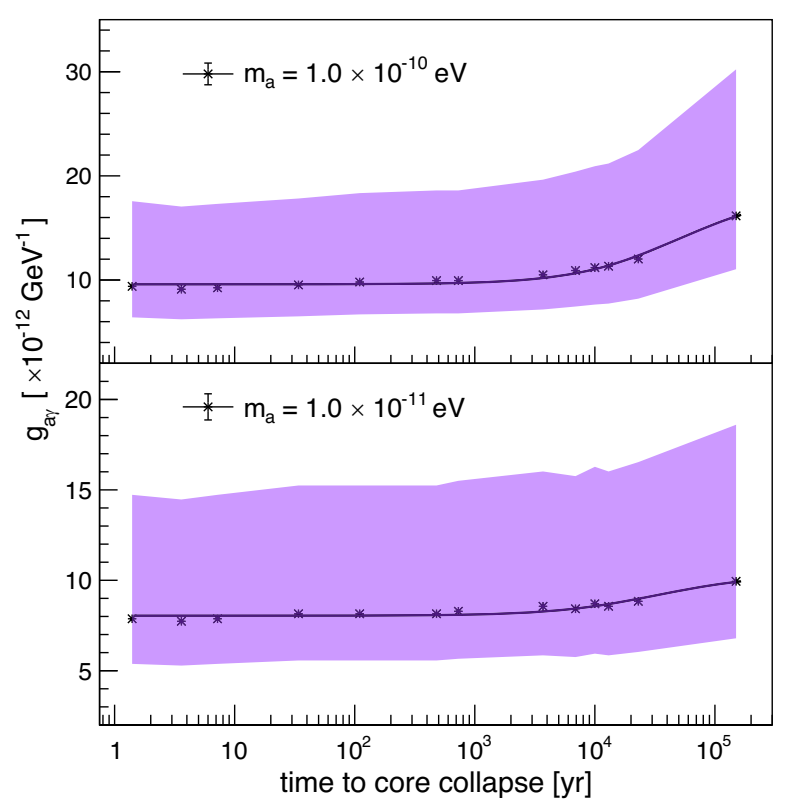

FIG. 4. Evolution on the derived 95\% C.L. upper limit of $g_{a \gamma}$ with remaining time until the core collapse for Betelgeuse. Results are shown for $m_{a}=1.0 \times 10^{-11}$ and $m_{a}=$ $1.0 \times 10^{-10} \mathrm{eV}$ assuming $B_{T}=1.4 \mu \mathrm{G}$ (more masses are shown in the Supplemental Material [25]). The solid black line shows the fitting. The width of the violet band indicates the uncertainty due to choice of $B_{T}$.

work while the width of the light red band reflects the uncertainty due to choice of stellar model and $B_{T}$. Using our most conservative assumptions $\left(t_{\mathrm{cc}}=1.55 \times 10^{5} \mathrm{yr}\right.$ and $B_{T}=0.4 \mu \mathrm{G}$ ), we set an upper limit of $g_{a \gamma}<1.8 \times$ $10^{-11} \mathrm{GeV}^{-1}$ for $m_{a}<3.5 \times 10^{-11} \mathrm{eV}$. In the scenario that predicts the highest ALP flux $\left(t_{\mathrm{cc}}=3.6 \mathrm{yr}\right.$ and $B_{T}=3.0 \mu \mathrm{G}$ ), we derive an upper limit of $g_{a \gamma}<5.2 \times$ $10^{-12} \mathrm{GeV}^{-1}$ for $m_{a}<5.5 \times 10^{-11} \mathrm{eV}$.

The uncertainty in our derived limit is dominated by our choice of $B_{T}$, since the ALP-photon conversion probability of Eq. (4) scales as $B_{T}^{2}$. The separate contributions to the uncertainty are illustrated in Fig. 4, which shows the evolution of our derived $g_{a \gamma}$ for different $t_{\mathrm{cc}}$. The solid black line is a fit to the $g_{a \gamma}$ derived for $B_{T}=1.4 \mu \mathrm{G}$, shown by the black points. The width of the magenta band then indicates the uncertainty due to our lower and upper bounds on $B_{T}$. The dependence on our assumed $B_{T}$ is further illustrated in Fig. S6, where we show our constraint in terms of $\mathrm{GeV}^{-1} \sqrt{\mu \mathrm{G}}$ for our range of $t_{\mathrm{cc}}$.

Conclusions and discussions.-Figure 3 shows the current theoretical and experimental ALP landscape. This includes the current constraints from CAST [10], ABRACADABRA-10 cm [57], and cavity experiments such as ADMX [56]. The yellow band indicates the preferred region in which the axion couples to quantum chromodynamics (QCD) and could be a solution to the strong- $C P$ problem [55], and the hatched band indicates the region where an ALP could explain the observed excess 
cooling of horizontal branch stars [58,59]. The limits derived in this work exceed those set by CAST by a factor of $\sim 3$ for $m_{a}<3.5 \times 10^{-11} \mathrm{eV}$, and are comparable in this low-mass space to the sensitivity of even next-generation experiments such as ALPS-II [22] and BabyIAXO [23,24].

Figure 1 compares our result to other astrophysical constraints in the low-mass ALP regime. Our most conservative limit exceeds the bounds from extragalactic supernovae [12] for $m_{a}<10^{-10} \mathrm{eV}$, and in our most optimistic ALP flux scenario our limit is comparable to that derived from the nonobservation of gamma rays from SN 1987A [11]. However, the supernova bounds have been questioned due to the modeling of core-collapse supernovae. For $m_{a}<10^{-11} \mathrm{eV}$, our limits are superseded by those derived from the lack of spectral variation observed by Chandra in the active galactic nucleus NGC 1275 [14] and in the core of M87 [16]. We caution, though, that more recent analysis shows that these results could be weakened by several orders of magnitude depending on the relative magnitude of the regular and turbulent intracluster magnetic fields that are assumed [15].

We emphasize that since each of these astrophysical constraints has unique sources of systematic error that may affect the final result, it is worthwhile to survey similar regions of parameter space with multiple techniques. The constraints presented here assume conversion in a homogeneous regular magnetic field, which allows for consistent comparison with previous astrophysical bounds, e.g., SN 1987A [11]. Although the magnetic structure of the Galaxy is certainly more complex, compared to more distant sources, the proximity of Betelgeuse should allow future observations to better constrain the relevant smallscale variations in magnetic structure, especially the ultralocal magnetic fields of the solar region which are not yet completely mapped (e.g., Refs. $[38,60,61])$. The combination of the novel ALP constraint presented here with multiple overlapping astrophysical constraints, each with separate modeling assumptions and uncertainties, builds confidence in the robustness of the exclusion of this corner of parameter space for low-mass ALPs.

We finally comment that a synergy between our astrophysical approach and direct ALP searches might lead to surprises and also unexpected benefits. Indeed, it might be that a future low-mass ALP experiment such as ABRACADABRA [62], DM-Radio [63], or IAXO [23] would discover an ALP in the region where optimistic assumptions on Galactic $B$-field and stellar model would have led to an exclusion from Betelgeuse. In this case one would come back to our original assumptions. Taking into account the typical uncertainty on the $B$ field one would give a lower limit on the time until the core collapse for Betelgeuse, as shown in Fig. 4 for an ALP mass and a coupling in the range $g_{a \gamma}=(5-30) \times 10^{-12} \mathrm{GeV}^{-1}$. Intriguingly ALPs might represent the only possibility to extract such information about the fate of Betelgeuse.
We thank D.R. Wik and S. Rossland for helpful discussions on the NUSTAR instrument background; J. L. Han and S. Zhang for discussions concerning the Galactic magnetic field in the direction of Betelgeuse; Benjamin Safdi, Christopher Dessert, John Beacom, Shunsaku Horiuchi, and Kenny C. Y. Ng for helpful comments and discussions. The NUSTAR observations described in this work were awarded under NASA Grant No. 80NSSC20K0031. The computational aspects of this work made extensive use of the following packages: SAOIMAGE DS9 distributed by the Smithsonian Astrophysical Observatory; the SCIPY ecosystem, particularly MATPLOTLIB and NUMPY; and ASTROPY, a communitydeveloped core PYTHON package for Astronomy. This research has made use of data and software provided by the High Energy Astrophysics Science Archive Research Center (HEASARC), which is a service of the Astrophysics Science Division at NASA/GSFC and the High Energy Astrophysics Division of the Smithsonian Astrophysical Observatory. We thank the NUSTAR Operations, Software and Calibration teams for support with the execution and analysis of these observations. This research made use of the NUSTAR Data Analysis Software (NuSTARDAS), jointly developed by the ASI Science Data Center (ASDC, Italy) and the California Institute of Technology (USA). M. X. and M. G. were supported by NASA Grant No. 80NSSC20K0031. O.S. has been supported by the Agenzia Spaziale Italiana (ASI) and the Instituto Nazionale di Astrofsica (INAF) under the Agreement No. 2017-14H.0 - attività di studio per la comunità scientifica di Astrofisica delle Alte Energie e Fisica Astroparticellare. A. M. is partially supported by the Italian Istituto Nazionale di Fisica Nucleare (INFN) through the "Theoretical Astroparticle Physics" project and by the research Grant No. 2017W4HA7S "NAT-NET: Neutrino and Astroparticle Theory Network" under the program PRIN 2017 funded by the Italian Ministero dell'Università e della Ricerca (MUR). B. G. was supported under NASA Contract No. NNG08FD60C.

*mjxiao@mit.edu

†kmperez@mit.edu

¥mgiannotti@barry.edu

§oscar.straniero@inaf.it

॥alessandro.mirizzi@ba.infn.it

"bwgref@srl.caltech.edu

**oachb@mit.edu

†mnynka@mit.edu

[1] L. Di Luzio, M. Giannotti, E. Nardi, and L. Visinelli, The landscape of QCD axion models, Phys. Rep. 870, 1 (2020).

[2] J. Jaeckel and A. Ringwald, The low-energy frontier of particle physics, Annu. Rev. Nucl. Part. Sci. 60, 405 (2010).

[3] G. Raffelt and L. Stodolsky, Mixing of the photon with low mass particles, Phys. Rev. D 37, 1237 (1988).

[4] P. Sikivie, Invisible axion search methods, arXiv:2003.02206. 
[5] I. G. Irastorza and J. Redondo, New experimental approaches in the search for axion-like particles, Prog. Part. Nucl. Phys. 102, 89 (2018).

[6] G. G. Raffelt, Astrophysical axion bounds diminished by screening effects, Phys. Rev. D 33, 897 (1986).

[7] A. Ayala, I. Domínguez, M. Giannotti, A. Mirizzi, and O. Straniero, Revisiting the Bound on Axion-Photon Coupling from Globular Clusters, Phys. Rev. Lett. 113, 191302 (2014).

[8] O. Straniero, A. Ayala, M. Giannotti, A. Mirizzi, and I. Dominguez, Axion-photon coupling: Astrophysical constraints, in Proceedings of the 11th Patras Workshop on Axions, WIMPs and WISPs (DESY, Hamburg, 2015), pp. 77-81.

[9] P. Carenza, O. Straniero, B. Döbrich, M. Giannotti, G. Lucente, and A. Mirizzi, Constraints on the coupling with photons of heavy axion-like-particles from Globular Clusters, Phys. Lett. B 809, 135709 (2020).

[10] V. Anastassopoulos et al. (CAST Collaboration), New CAST limit on the axion-photon interaction, Nat. Phys. 13, 584 (2017).

[11] A. Payez, C. Evoli, T. Fischer, M. Giannotti, A. Mirizzi, and A. Ringwald, Revisiting the SN1987A gamma-ray limit on ulltralight axion-like particles, J. Cosmol. Astropart. Phys. 02 (2015) 006.

[12] M. Meyer and T. Petrushevska, Search for AxionlikeParticle-Induced Prompt Gamma-Ray Emission from Extragalactic Core-Collapse Supernovae with the Fermi Large Area Telescope, Phys. Rev. Lett. 124, 231101 (2020).

[13] D. J. Forrest et al., The gamma ray spectrometer for the solar maximum mission, Sol. Phys. 65, 15 (1980).

[14] C. S. Reynolds, M. C. David Marsh, H. R. Russell, A. C. Fabian, R. Smith, F. Tombesi, and S. Veilleux, Astrophysical limits on very light axion-like particles from chandra grating spectroscopy of NGC 1275, Astrophys. J. 890, 59 (2019).

[15] M. Libanov and S. Troitsky, On the impact of magnetic-field models in galaxy clusters on constraints on axion-like particles from the lack of irregularities in high-energy spectra of astrophysical sources, Phys. Lett. B 802, 135252 (2020).

[16] M. C. David Marsh, H. R. Russell, A. C. Fabian, B. P. McNamara, P. Nulsen, and C. S. Reynolds, A new bound on axion-like particles, J. Cosmol. Astropart. Phys. 12 (2017) 036.

[17] E. D. Carlson, Pseudoscalar conversion and X-rays from stars, Phys. Lett. B 344, 245 (1995).

[18] J. Posson-Brown, V. L. Kashyap, D. O. Pease, and J. J. Drake, Invisible giant: Chandra's limits on X-rays from betelgeuse, arXiv:astro-ph/0606387.

[19] C. Dessert, J. W. Foster, and B. R. Safdi, X-ray Searches for Axions from Super Star Clusters, Phys. Rev. Lett. 125, 261102 (2020).

[20] G. M. Harper, A. Brown, and E. F. Guinan, A New VLAhipparcos distance to betelgeuse and its implications, Astron. J. 135, 1430 (2008).

[21] G. M. Harper, A. Brown, E. F. Guinan, E. O'Gorman, A. M. S. Richards, P. Kervella, and L. Decin, An updated 2017 astrometric solution for betelgeuse, Astron. J. 154, 11 (2017).

[22] R. Bähre et al., Any light particle search II-Technical Design Report, J. Instrum. 8, T09001 (2013).
[23] E. Armengaud et al. (IAXO Collaboration), Physics potential of the international axion observatory (IAXO), J. Cosmol. Astropart. Phys. 06 (2019) 047.

[24] P. Di Vecchia, M. Giannotti, M. Lattanzi, and A. Lindner, Round table on axions and axion-like particles, Proc. Sci. Confinement2018 (2019) 034 [arXiv:1902.06567].

[25] See Supplemental Material at http://link.aps.org/ supplemental/10.1103/PhysRevLett.126.031101 for contains additional results and explanations that support the results presented in the main Letter. We provide further details regarding the Betelgeuse stellar models, NuSTAR instrument background modeling, and cross-check of our results with Chandra observation of Betelgeuse, and present additional results of $B_{T}$ depended ALP-photon coupling and the evolution of ALP-photon coupling for more ALP masses. Refs. [26-34] are included in this Supplemental Material.

[26] A.C. Fabian and X. Barcons, The origin of the X-ray background, Annu. Rev. Astron. Astrophys. 30, 429 (1992).

[27] M. Revnivtsev, M. Gilfanov, R. Sunyaev, K. Jahoda, and C. Markwardt, The spectrum of the cosmic X-ray background observed by RTXE/PCA, Astron. Astrophys. 411, 329 (2003).

[28] A. Fruscione, J. C. McDowell, G. E. Allen et al., CIAO: Chandra's data analysis system, Proc. SPIE Int. Soc. Opt. Eng. 6270, 62701V (2006).

[29] S. Andriamonje et al. (CAST Collaboration), An improved limit on the axion-photon coupling from the CAST experiment, J. Cosmol. Astropart. Phys. 04 (2007) 010.

[30] T. Le Bertre, L. D. Matthews, E. Gérard, and Y. Libert, Discovery of a detached H I gas shell surrounding $\alpha$ Orionis, Mon. Not. R. Astron. Soc. 422, 3433 (2012).

[31] G. Perrin, S. T. Ridgway, V. Coudé du Foresto, B. Mennesson, W. A. Traub, and M. G. Lacasse, Interferometric observations of the supergiant stars $\alpha$ Orionis and $\alpha$ Herculis with FLUOR at IOTA, Astron. Astrophys. 418, 675 (2004).

[32] D. L. Lambert, J. A. Brown, K. H. Hinkle, and Johnson, Carbon, nitrogen and oxygem abundances in Betelgeuse, Astrophys. J. 284, 223 (1984).

[33] G. Meynet, L. Haemmerlé, S. Ekström, C. Georgy, J. Groh, and A. Maeder, The past and future evolution of a star like Betelgeuse, in EAS Publications Series, EAS Publications Series, Vol. 60, edited by P. Kervella, T. Le Bertre, and G. Perrin (EDP Sciences, Les Ulis, 2013), pp. 17-28.

[34] M. M. Dolan, G. J. Mathews, D. D. Lam, N. Quynh Lan, G. J. Herczeg, and D. S. P. Dearborn, Evolutionary tracks for betelgeuse, Astrophys. J. 819, 7 (2016).

[35] O. Straniero, I. Dominguez, L. Piersanti, M. Giannotti, and A. Mirizzi, The initial mass-final luminosity relation of type II supernova progenitors: Hints of new physics?, Astrophys. J. 881, 158 (2019).

[36] J. L. Han, Observing interstellar and intergalactic magnetic fields, Annu. Rev. Astron. Astrophys. 55, 111 (2017).

[37] R. Jansson and G. R. Farrar, A new model of the galactic magnetic field, Astrophys. J. 757, 14 (2012).

[38] J. Xu and J. L. Han, Magnetic fields in the solar vicinity and in the Galactic halo, Mon. Not. R. Astron. Soc. 486, 4275 (2019).

[39] M. C. Beck, A. M. Beck, R. Beck, K. Dolag, A. W. Strong, and P. Nielaba, New constraints on modelling the random 
magnetic field of the MW, J. Cosmol. Astropart. Phys. 05 (2016) 056.

[40] V. Pelgrims, K. Ferrière, F. Boulanger, R. Lallement, and L. Montier, Modeling the magnetized Local Bubble from dust data, Astron. Astrophys. 636, A17 (2020).

[41] N. Bassan, A. Mirizzi, and M. Roncadelli, Axion-like particle effects on the polarization of cosmic high-energy gamma sources, J. Cosmol. Astropart. Phys. 05 (2010) 010.

[42] J. M. Cordes, and T. J. W. Lazio, NE2001.I. A New Model for the Galactic Distribution of Free Electrons and its Fluctuations, arXiv:astro-ph/0207156.

[43] L. Harvey-Smith, G. J. Madsen, and B. M. Gaensler, Magnetic fields in large diameter HII regions revealed by the faraday rotation of compact extragalactic radio sources, Astrophys. J. 736, 83 (2011).

[44] F. A. Harrison et al., The nuclear spectroscopic telescope array (NuSTAR) high-energy X-ray mission, Astrophys. J. 770, 103 (2013).

[45] K. K. Madsen et al., Calibration of the NuSTAR high energy focusing X-ray telescope, Astrophys. J. Suppl. Ser. 220, 8 (2015).

[46] K. Perez, R. Krivonos, and D. R. Wik, The galactic bulge diffuse emission in broadband X-rays with NuSTAR, Astrophys. J. 884, 153 (2019).

[47] B. M. Roach, K. C. Y. Ng, K. Perez, J. F. Beacom, S. Horiuchi, R. Krivonos, and D. R. Wik, NuSTAR tests of sterile-neutrino dark matter: New galactic bulge observations and combined impact, Phys. Rev. D 101, 103011 (2020).

[48] F. van Leeuwen, Validation of the new hipparcos reduction, Astron. Astrophys. 474, 653 (2007).

[49] I. N. Evans et al., The chandra source catalog, Astrophys. J. Suppl. Ser. 189, 37 (2010).

[50] D. R. Wik et al., NuSTAR observations of the bullet cluster: Constraints on inverse compton emission, Astrophys. J. 792, 48 (2014).

[51] R. Brun and F. Rademakers, ROOT: An object oriented data analysis framework, Nucl. Instrum. Methods Phys. Res., Sect. A 389, 81 (1997).
[52] T. Junk, Confidence level computation for combining searches with small statistics, Nucl. Instrum. Methods Phys. Res., Sect. A 434, 435 (1999).

[53] G. Cowan, K. Cranmer, E. Gross, and O. Vitells, Asymptotic formulae for likelihood-based tests of new physics, Eur. Phys. J. C 71, 1554 (2011); 73, 2501(E) (2013).

[54] G. J. Feldman and R. D. Cousins, A unified approach to the classical statistical analysis of small signals, Phys. Rev. D 57, 3873 (1998).

[55] L. Di Luzio, F. Mescia, and E. Nardi, Redefining the Axion Window, Phys. Rev. Lett. 118, 031801 (2017).

[56] T. Braine et al. (ADMX Collaboration), Extended Search for the Invisible Axion with the Axion Dark Matter Experiment, Phys. Rev. Lett. 124, 101303 (2020).

[57] J. L. Ouellet et al., First Results from ABRACADABRA$10 \mathrm{~cm}$ : A Search for Sub- $\mu \mathrm{eV}$ Axion Dark Matter, Phys. Rev. Lett. 122, 121802 (2019).

[58] M. Giannotti, I. Irastorza, J. Redondo, and A. Ringwald, Cool WISPs for stellar cooling excesses, J. Cosmol. Astropart. Phys. 05 (2016) 057.

[59] M. Giannotti, I. G. Irastorza, J. Redondo, A. Ringwald, and K. Saikawa, Stellar recipes for axion hunters, J. Cosmol. Astropart. Phys. 10 (2017) 010.

[60] M. Salvati, The local Galactic magnetic field in the direction of Geminga, Astron. Astrophys. 513, A28 (2010).

[61] P. C. Frisch, B-G Andersson, A. Berdyugin, V. Piirola, R. DeMajistre, H. O. Funsten, A. M. Magalhaes, D. B. Seriacopi, D. J. McComas, N. A. Schwadron, J. D. Slavin, and S. J. Wiktorowicz, The interstellar magnetic field close to the SUN. II, Astrophys. J. 760, 106 (2012).

[62] J.L. Ouellet et al., Design and implementation of the ABRACADABRA-10 cm axion dark matter search, Phys. Rev. D 99, 052012 (2019).

[63] M. Silva-Feaver et al., Design overview of DM radio pathfinder experiment, IEEE Trans. Appl. Supercond. 27, 1 (2017). 